\title{
Safety and Feasibility of Intravenous Paracetamol for Patent Ductus Arteriosus in Indomethacin-/Ibuprofen-Resistant or -Contraindicated Preterm Infants: A Case Series
}

\author{
Ayumi Oshima, MD1 Shun Matsumura, MD ${ }^{1}$ Ayaka Iwatani, MD 1 Machiko Morita, MD 1 \\ Sumie Fujinuma, MD ${ }^{1}$ Yukiko Motojima, MD ${ }^{1}$ Kosuke Tanaka, MD ${ }^{1}$ Satoshi Masutani, MD, PhD ${ }^{1}$ \\ Kazuhiko Kabe, $\mathrm{MD}^{1}$ Keiko Ueda, $\mathrm{MD}^{2}$ Fumihiko Namba, MD, $\mathrm{PhD}^{1}$

\footnotetext{
${ }^{1}$ Department of Pediatrics, Saitama Medical Center, Saitama Medical University, Saitama, Japan

2 Regulatory Strategy Division, Clinical Research Support Center, The University of Tokyo Hospital, Tokyo, Japan
} \\ Address for correspondence Fumihiko Namba, MD, PhD, Department \\ of Pediatrics, Saitama Medical Center, Saitama Medical University, \\ 1981 Kamoda, Kawagoe, Saitama 350-8550, Japan \\ (e-mail: nambaf@saitama-med.ac.jp).
}

\begin{abstract}
Keywords

- ibuprofen

- indomethacin

- paracetamol

- patent ductus arteriosus

- premature infant

- surgical closure

Background Although indomethacin and ibuprofen are the standard treatments for hemodynamically significant patent ductus arteriosus (hsPDA), they are associated with renal impairment and gastrointestinal complications. Paracetamol for hsPDA closure does not provoke a peripheral vasoconstrictive effect and seems to have effects similar to those of indomethacin and ibuprofen. We have previously reported the safety of low-dose $(7.5 \mathrm{mg} / \mathrm{kg})$ intravenous paracetamol for preterm infants with hsPDA, who were indomethacin-resistant or-contraindicated but did not affect the need for surgical PDA ligation. However, reports considering the use of higher-dose $(15 \mathrm{mg} / \mathrm{kg})$ paracetamol for hsPDA have not been published in Japan.

Cases In 16 premature infants in whom indomethacin or ibuprofen was contraindicated or ineffective, $15 \mathrm{mg} / \mathrm{kg}$ of paracetamol was intravenously administered every 6 hours for 3 days after obtaining parental consent. hsPDA closure or narrowing was observed in 14 infants (88\%), with the need for surgical closure totally avoided in nine cases (56\%). High plasma paracetamol levels were observed in three cases. No paracetamol-related side effects or adverse events were reported.

Conclusion The intravenous administration of higher dose paracetamol was safe and feasible in premature infants with hsPDA. Future clinical trials to explore the optimized dose and timing of administration are needed.
\end{abstract}

\section{Background}

Ductus arteriosus (DA) is an essential blood vessel for fetal circulation and development. In most term infants, DA closes spontaneously within 1 to 2 days after birth due to the loss of the prostaglandin supply from the placenta and the rise of arterial oxygen pressure. ${ }^{1}$ In contrast, DA often does not close spontaneously in preterm infants, including especially very-low-birth-weight infants (VLBWIs), due to the poor responsiveness to arterial oxygen pressure and the high responsiveness to prostaglandin inherent in this population. ${ }^{2,3}$ It is well known that hemodynamically significant received

September 10, 2019

accepted after revision

October 30, 2019
DOI https://doi.org/

10.1055/s-0040-1702945. ISSN 2157-6998.
Copyright $\odot 2020$ by Thieme Medical Publishers, Inc., 333 Seventh Avenue, New York, NY 10001, USA. Tel: +1(212) 760-0888.
License terms

(c) (1) $\ominus$ (\$) 
patent ductus arteriosus (hsPDA) increases the risk of intraventricular hemorrhage (IVH), necrotizing enterocolitis, and bronchopulmonary dysplasia. ${ }^{4-6}$ In Japan, the cyclooxygenase inhibitor-indomethacin-has been used as standard therapy for hsPDA. In addition, another cyclooxygenase inhibitor-ibuprofen-has been available for application in cases of hsPDA since June 2018. However, because of their side effects such as acute kidney injury and gastrointestinal perforation, the introduction of other novel treatments for hsPDA with fewer side effects is required. ${ }^{6}$

Paracetamol has recently been reported to have the same effects on DA closure with fewer side effects as those of indomethacin and ibuprofen observed during the treatment for hsPDA. ${ }^{7-12}$ We have previously reported a case series of three patients in Japan treated with low-dose intravenous paracetamol $(7.5 \mathrm{mg} / \mathrm{kg}$, given every 6 hours for 3 days $)$ for preterm infants with hsPDA, who were indomethacinresistant or -contraindicated. A temporary DA closure was observed in two of the three infants without any side effects. However, all three infants eventually required surgical closure. ${ }^{13}$ This treatment failure was thought to be due to an inadequate dose of paracetamol, given that a higher dose ( $15 \mathrm{mg} / \mathrm{kg}$, given every 6 hours for 3 days) has been used in most studies, elsewhere in which degree of the efficacy of paracetamol on DA closure was observed. ${ }^{7-11}$ Therefore, in the present research, we increased the dose of paracetamol up to $15 \mathrm{mg} / \mathrm{kg} /$ dose for evaluation in 16 preterm infants who were indomethacin/ibuprofen-resistant or -contraindicated and examined the safety and feasibility outcomes.

\section{Methods}

About 16 VLBWIs (birth weight $<1,500 \mathrm{~g}$ ) with hsPDA born at Saitama Medical Center, Saitama Medical University from July 1, 2017 to March 31, 2019 were included in this research. hsPDA was determined by each physician using echocardiography and ultrasonography of the brain and abdomen. These patients showed resistance or contraindications, such as acute renal failure, to indomethacin and/or ibuprofen. In each patient, $15 \mathrm{mg} / \mathrm{kg} /$ dose of paracetamol (Acelio Intravenous Injection; TERUMO Co. Ltd., Tokyo, Japan) was administered every 6 hours for 3 days as one course. Arterial duct diameter, left atrial aortic root ratio, and end-diastolic flow velocity of the left pulmonary artery were subsequently evaluated using ultrasonography by the physician. Physical examinations; vital signs; and blood tests including creatinine $(\mathrm{Cr})$, aspartate aminotransferase (AST), alanine aminotransferase (ALT), and acetaminophen blood levels were performed to evaluate the presence of adverse events. Acute renal failure was defined as serum $\mathrm{Cr}$ level $>1.5 \mathrm{mg} / \mathrm{dL}$. Elevated AST and ALT levels were defined as $>100$ and $>280 \mathrm{U} / \mathrm{L}$, respectively. This study was covered by the clinical trial insurance program, and was approved by the institutional review board of Saitama Medical Center, Saitama Medical University (approval no. 1656-III). Parental consent was obtained before paracetamol administration.

\section{Results}

About 16 patients were included in this study (-Table 1). The median gestational age was 27.6 weeks (range: 23.0-29.4 weeks) and the median birth weight was $913 \mathrm{~g}$ (range: 475-1,423 g). Paracetamol was administered because of resistance to indomethacin or ibuprofen in seven cases and the presence of acute kidney injury in ten cases (one case had both findings). The median starting date of paracetamol administration was a day 10 after birth (range: days 2-36), and the median duration of paracetamol administration was 6 days (range: $3-9$ days). The initial median DA diameter was $1.3 \mathrm{~mm}$ (range: $0.8-2.2 \mathrm{~mm}$ ), and the initial median left atrial aortic root ratio was 1.66 (range: 0.87-2.08). Among the 16 cases, paracetamol treatment was effective in 14 cases (88\%), with DA closure or narrowing observed, while two cases (13\%) were refractory to the therapy. Surgical closure of PDA was required in seven cases (44\%), and the DA remained open at discharge and was followed up on an outpatient basis in one case $(6 \%)$.

In all 16 cases, no adverse events-including elevated AST or ALT levels, hypothermia $\left(<36^{\circ} \mathrm{C}\right.$ body temperature), or the need for a higher incubator temperature-were observed after paracetamol administration. Plasma paracetamol levels were measured in ten cases (-Fig. 1). In two (cases 7 and 13) of the ten cases, the third course of paracetamol administration was avoided because relatively high plasma paracetamol levels (64.3 and $97.7 \mu \mathrm{g} / \mathrm{mL}$, respectively) were observed at 6 hours after the earlier two courses of paracetamol treatments. In addition, in one case (case 16), plasma paracetamol level of $116.7 \mu \mathrm{g} / \mathrm{mL}$ was observed 4 hours after the first course of treatment, so the second course was discontinued.

In case 1 , colostomy was performed on day 5 after the birth for congenital intestinal atresia, and ascites could not be controlled thereafter, resulting in death on day 64 . Cases 2 and 3 were monochorionic diamniotic twins with twin-to-twin transfusion syndrome, and both patients had periventricular leukomalacia from birth. Case 14 developed IVH on day 1 after birth with circulatory failure due to intrauterine infection. Case 16 was born with fetal heart failure and fetal hydrops, and had IVH and intracerebral hemorrhage.

\section{Discussion}

In this case series, we confirmed the safety and feasibility of higher-dose intravenous paracetamol $(15 \mathrm{mg} / \mathrm{kg}$ given every 6 hours for 3 days) for VLBWIs with hsPDA who were indomethacin/ibuprofen-resistant or -contraindicated in Japan, similar to that previously observed in patients with low-dose intravenous paracetamol $(7.5 \mathrm{mg} / \mathrm{kg}$, given every 6 hours for 3 days). ${ }^{13}$

In our previous study performed using low-dose intravenous paracetamol ( $7.5 \mathrm{mg} / \mathrm{kg}$ given every 6 hours for 3 days), temporary DA closure was observed in two of three (67\%) cases with hsPDA and no short-term adverse events occurred during the provision of pharmacological treatment in any cases. However, our protocol of applying low-dose intravenous paracetamol ultimately could not avoid the need for surgical 


\begin{tabular}{|c|c|c|c|c|c|c|c|c|c|c|c|c|c|c|c|c|}
\hline 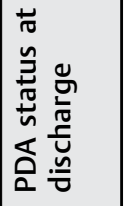 & $\begin{array}{l}\stackrel{\Xi}{凶} \\
\tilde{O} \\
\text { U. }\end{array}$ & $\begin{array}{l}\stackrel{D}{\cup} \\
\stackrel{\tilde{O}}{U}\end{array}$ & $\left|\begin{array}{l}\tilde{U} \\
\tilde{U} \\
\underline{U}\end{array}\right|$ & $\left|\begin{array}{l}\tilde{D} \\
\tilde{U} \\
\mathcal{U}\end{array}\right|$ & $\begin{array}{l}\tilde{D} \\
\tilde{U} \\
0 \\
U\end{array}$ & 㐫 & 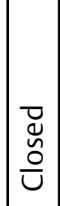 & $\left|\begin{array}{l}\tilde{U} \\
\tilde{U} \\
\underline{U}\end{array}\right|$ & $\left|\begin{array}{l}\tilde{D} \\
\tilde{U} \\
\mathcal{U}\end{array}\right|$ & $\left|\begin{array}{c}\tilde{U} \\
\tilde{O} \\
\cup\end{array}\right|$ & $\begin{array}{l}\bar{D} \\
\stackrel{\Xi}{u} \\
0 \\
\text { U }\end{array}$ & 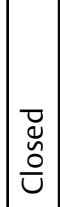 & $\begin{array}{l}\tilde{O} \\
\tilde{U} \\
\text { U }\end{array}$ & $\left|\begin{array}{l}\tilde{D} \\
\tilde{\Delta} \\
\tilde{U}\end{array}\right|$ & $\left|\begin{array}{l}\tilde{U} \\
\tilde{U} \\
\tilde{U}\end{array}\right|$ & 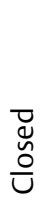 \\
\hline 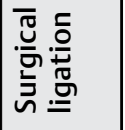 & + & 1 & 1 & + & 1 & 1 & + & + & 1 & 1 & 1 & 1 & 1 & + & + & + \\
\hline 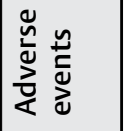 & 1 & 1 & 1 & 1 & 1 & 1 & 1 & 1 & 1 & 1 & 1 & 1 & 1 & 1 & 1 & 1 \\
\hline 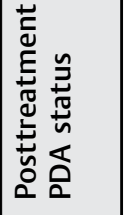 & 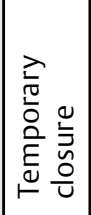 & 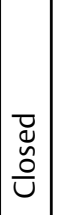 & $\mid \begin{array}{l}\tilde{D} \\
\tilde{\omega} \\
\tilde{O} \\
U\end{array}$ & 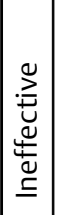 & 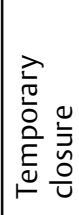 & 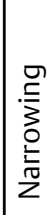 & 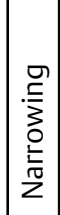 & 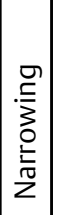 & 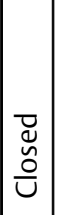 & $\left|\begin{array}{c}\tilde{D} \\
\tilde{u} \\
\tilde{U} \\
\end{array}\right|$ & 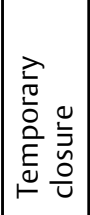 & $\begin{array}{l} \\
\tilde{D} \\
\tilde{U} \\
\stackrel{\cup}{U}\end{array}$ & 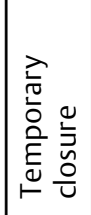 & 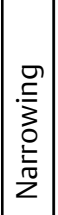 & 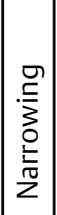 & 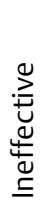 \\
\hline 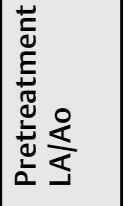 & $\stackrel{\curvearrowright}{\check{2}}$ & $\begin{array}{l}\text { ğ } \\
r\end{array}$ & $\left|\begin{array}{l}\infty \\
0 \\
- \\
-\end{array}\right|$ & פִ & $\stackrel{m}{r}$ & $\begin{array}{l}\infty \\
\stackrel{\infty}{\sim} \\
\sim\end{array}$ & 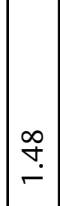 & 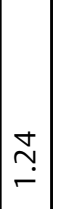 & $\mid \begin{array}{l}\tilde{\varphi} \\
-\end{array}$ & $\tilde{m}$ & $\mid \begin{array}{l}\infty \\
\infty \\
- \\
-\end{array}$ & $\begin{array}{l}0 \\
\infty \\
0\end{array}$ & กิ & $\stackrel{ }{\stackrel{P}{-}}$ & 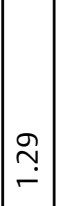 & $\stackrel{m}{\circ}$ \\
\hline 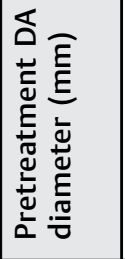 & $\stackrel{\varphi}{r}$ & $\stackrel{m}{r}$ & $\sim$ & - & $\stackrel{\text { ำ }}{r}$ & $\bar{i}$ & $\stackrel{\sim}{-}$ & $\begin{array}{l}0 \\
-\end{array}$ & $\stackrel{\sim}{\sim}$ & 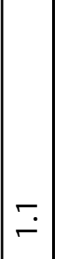 & 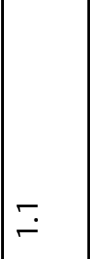 & $\begin{array}{l}\infty \\
\dot{0}\end{array}$ & $\stackrel{\sim}{r}$ & $\sim$ & $\stackrel{\sim}{\sim}$ & $\stackrel{9}{-}$ \\
\hline 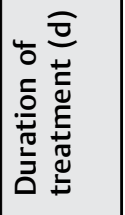 & 6 & $m$ & $m$ & $m$ & $m$ & 6 & 6 & 6 & 6 & a & $a$ & $m$ & $m$ & $\sigma$ & $m$ & $\sigma$ \\
\hline 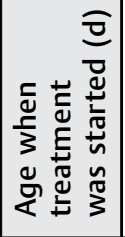 & $\nabla$ & $\sim$ & $m$ & $\stackrel{\curvearrowright}{\sim}$ & 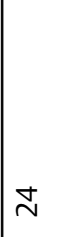 & $\stackrel{\llcorner}{\sim}$ & in & $a$ & 6 & $\infty$ & $\stackrel{+}{+}$ & $\begin{array}{l}0 \\
m\end{array}$ & 6 & $\mp$ & $\stackrel{\Perp}{\sim}$ & $m$ \\
\hline 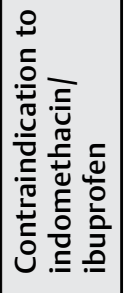 & 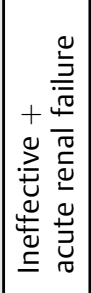 & 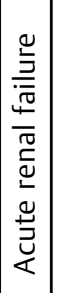 & 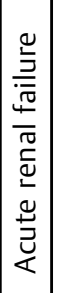 & 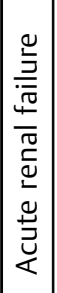 & 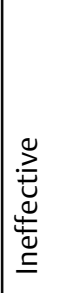 & 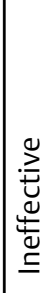 & 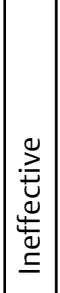 & 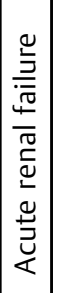 & 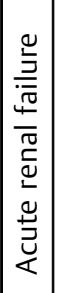 & 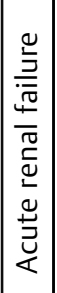 & 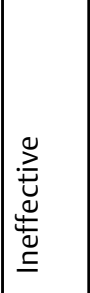 & 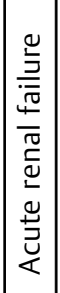 & 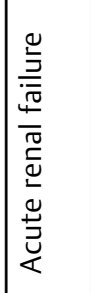 & 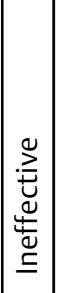 & 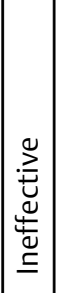 & 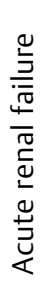 \\
\hline ত্ & ֻูกิ & $\begin{array}{l}m \\
\stackrel{n}{0} \\
- \\
-\end{array}$ & $\overline{\mathbb{N}}$ & $\begin{array}{c}0 \\
m \\
0 \\
- \\
-\end{array} \mid$ & $\begin{array}{l}\hat{m} \\
\underline{\underline{n}} \\
\stackrel{n}{-}\end{array}$ & 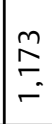 & م̊ & $\stackrel{\operatorname{Ln}}{\stackrel{\sim}{\gamma}}$ & 용 & $\bar{n}$ & $\underset{\infty}{\stackrel{D}{N}}$ & เૂ & 占 & \begin{tabular}{l} 
Ln \\
\multicolumn{0}{c}{} \\
-
\end{tabular} & $\left|\begin{array}{l}m \\
\infty \\
0 \\
- \\
-\end{array}\right|$ & 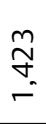 \\
\hline$\frac{\frac{5}{3}}{\frac{\pi}{3}}$ & 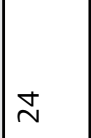 & $\stackrel{\infty}{\sim}$ & $\stackrel{\infty}{\sim}$ & $\stackrel{\stackrel{\sim}{\curvearrowleft}}{\sim}$ & ป & ป & $\stackrel{\bullet}{\sim}$ & $\stackrel{m}{\sim}$ & $\stackrel{\infty}{\sim}$ & $\stackrel{m}{\sim}$ & $\stackrel{\infty}{\sim}$ & $\stackrel{\searrow}{\sim}$ & $\cong$ & $\lesssim$ & $\stackrel{\infty}{\sim}$ & $\stackrel{\infty}{\sim}$ \\
\hline ஸे & - & $\sim$ & $m$ & $\nabla$ & in & 6 & $r$ & $\infty$ & $a$ & $\stackrel{\circ}{-}$ & $\mp$ & $\simeq$ & $\stackrel{m}{r}$ & $\stackrel{\Xi}{\longleftarrow}$ & $\stackrel{\llcorner}{\leftarrow}$ & 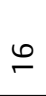 \\
\hline
\end{tabular}




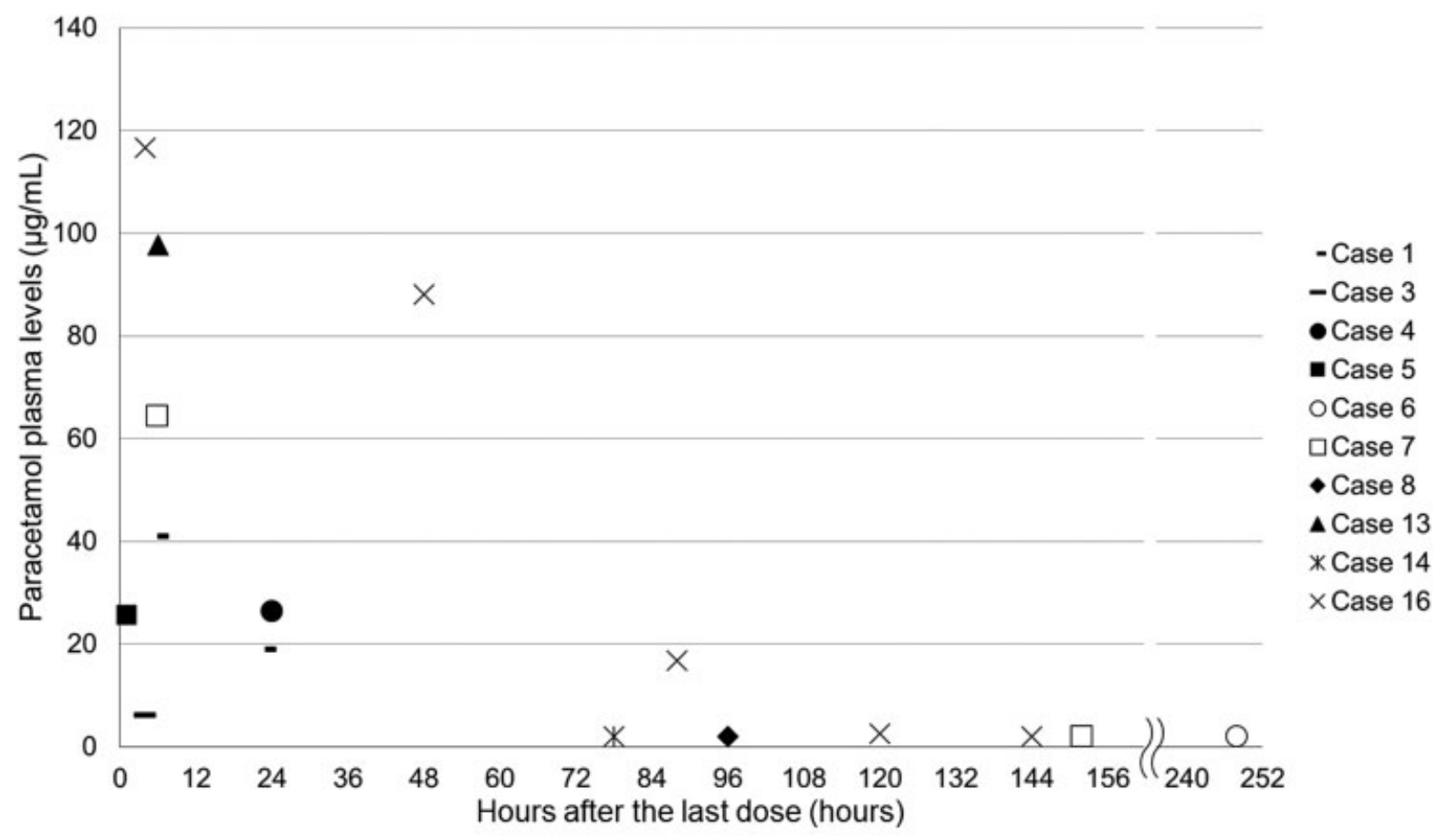

Fig. 1 Plasma paracetamol levels. The plasma paracetamol levels were measured in ten cases. The $x$-axis shows hours after the last dose of paracetamol (hours) and the $y$-axis shows plasma paracetamol levels $(\mu \mathrm{g} / \mathrm{mL})$.

treatment (avoidance rate of surgical ligation: $0 \%$ ). In contrast, the presently discussed pharmacological treatment approach with higher-dose intravenous paracetamol $(15 \mathrm{mg} / \mathrm{kg}$ given every 6 hours for 3 days) increased both the success of DA closure or narrowing $(14 / 16 ; 88 \%)$ and avoidance of surgical PDA ligation $(9 / 16 ; 56 \%)$ as compared with the low-dose paracetamol regimen. In a systematic review, the analysis of uncontrolled studies suggested that as compared with the use of low-dose paracetamol (30-60 mg/kg/day), the use of higher doses $(60 \mathrm{mg} / \mathrm{kg} /$ day) appears unnecessary because of no significant differences in DA closure rate between two doses. ${ }^{10}$ However, this result should be interpreted with caution considering the high proportion of subjects with a gestational age of more than 28 weeks treated with low doses. Therefore, higher-dose (15 mg/kg/dose) paracetamol should be used in further clinical trials involving patients with PDA who have resistance or contraindications to indomethacin and/or ibuprofen.

Of note, paracetamol clearance is lower in neonates than in children and adults. After metabolic conversion, conjugation with glucuronic acid and sulfate, paracetamol is subsequently eliminated via the renal route. In the present study, the plasma paracetamol levels were measured in ten cases. With acute ingestions of paracetamol, the Rumack-Mathews nomogram is a valuable tool to use to assess the risk of hepatotoxicity. This nomogram was originally constructed in the 1970s as a method to discriminate those patients who were more likely to suffer hepatotoxicity and those who were not. A line between $200 \mathrm{mg} / \mathrm{mL}$ at 4 hours after ingestion and $25 \mathrm{mg} / \mathrm{mL}$ at 16 hours, known as the "200 line" defined the group at risk. ${ }^{14}$ Conversely, a parallel line at $150 \mathrm{mg} / \mathrm{mL}$ at 4 hours-also known as the treatment line or "150 line"-is the treatment line most commonly used in the United States. ${ }^{15}$ Although no paracetamol-related adverse eventsincluding elevated AST or ALT levels-were observed in any patients in this research, another course of paracetamol administration was avoided because of high plasma paracetamol levels in three of ten cases in which plasma paracetamol levels were measured. All three cases had circulatory failure, oliguria, or elevated serum $\mathrm{Cr}$ levels before and during intravenous paracetamol treatment. Therefore, we stress that plasma paracetamol levels should be monitored in patients who are at a high risk for circulatory and/or renal failure.

We determine that good feasibility and short-term safety were present during pharmacological treatment with intravenous paracetamol in our research. However, it has been reported that the long-term administration of paracetamol to the pregnant mother is implicated in the development of autism or autism spectrum disorder in children. ${ }^{16}$ In contrast, however, one study reported that there were no differences in neurodevelopmental outcomes at 18 to 24 months, including on the Bayley Scales of Infant Development, second edition; the Mental Developmental Index; and the Psychomotor Developmental Index, with recommended adaptations suggested for visual and auditory impairments in preterm or low-birth weight infants exposed to paracetamol as compared with ibuprofen for PDA. ${ }^{17}$ Importantly, because the quality of evidence of this study is low, long-term follow-up to at least 18 to 24 months after birth must be incorporated in any studies of paracetamol in the newborn population, including our previous and present case series.

In our current case series, several limitations exist. (1) Being a descriptive study with a small number of patients 
and with the absence of a control group weakens our study, especially in the presence of several published randomized controlled pilot studies suggesting the efficacy and safety of paracetamol for hsPDA. ${ }^{8-11}$ However, those studies also included a limited number of cases. Therefore, reporting a case series still has some degree of enhancement to the quality of evidence as part of a cumulative data. (2) We confirmed the treatment's safety and feasibility with the anticipated results and a lack of serious adverse reactions in small populations whose treatment with paracetamol were determined by physician's discretion. To estimate the efficacy of this treatment in hsPDA patients, randomized control trials are warranted in the future.

\section{Conclusion}

This is the first report in Japan describing the safety and feasibility of treatment with higher dose intravenous paracetamol for preterm infants with hsPDA in whom indomethacin or ibuprofen was contraindicated or ineffective. Plasma paracetamol levels should be monitored in higher risk patients with circulatory and/or renal failure. Further follow-up study among these subjects and the conduction of prospective clinical trials such as randomized control trials are needed to evaluate the long-term prognosis and efficacy of intravenous paracetamol for hsPDA closure.

Funding

Saitama Medical Center Internal Research Grant for Young Physician Scientists (grant/award number: “30-F-1-18”).

\section{Conflict of Interest}

None declared.

\section{Acknowledgment}

This work was supported by the Saitama Medical Center Internal Research Grant for Young Physician Scientists (grant no. 30-F-1-18). The authors have no affiliations with or involvement in any organization or entity with any financial interest, or nonfinancial interest in the subject matter or materials discussed in this manuscript.

\section{References}

1 Gentile R, Stevenson G, Dooley T, Franklin D, Kawabori I, Pearlman A. Pulsed Doppler echocardiographic determination of time of ductal closure in normal newborn infants. J Pediatr 1981;98(03): 443-448
2 Clyman RI, Couto J, Murphy GM. Patent ductus arteriosus: are current neonatal treatment options better or worse than no treatment at all? Semin Perinatol 2012;36(02):123-129

3 Liu H, Manganiello V, Waleh N, Clyman RI. Expression, activity, and function of phosphodiesterases in the mature and immature ductus arteriosus. Pediatr Res 2008;64(05):477-481

4 Itabashi K, Ohno T, Nishida $\mathrm{H}$. Indomethacin responsiveness of patent ductus arteriosus and renal abnormalities in preterm infants treated with indomethacin. J Pediatr 2003;143(02): 203-207

5 Hamrick SE, Hansmann G. Patent ductus arteriosus of the preterm infant. Pediatrics 2010;125(05):1020-1030

6 Johnston PG, Gillam-Krakauer M, Fuller MP, Reese J. Evidencebased use of indomethacin and ibuprofen in the neonatal intensive care unit. Clin Perinatol 2012;39(01):111-136

7 Oncel MY, Yurttutan S, Degirmencioglu H, et al. Intravenous paracetamol treatment in the management of patent ductus arteriosus in extremely low birth weight infants. Neonatology 2013;103(03):166-169

8 Oncel MY, Yurttutan S, Erdeve O, et al. Oral paracetamol versus oral ibuprofen in the management of patent ductus arteriosus in preterm infants: a randomized controlled trial. J Pediatr 2014;164 (03):510-4.e1

9 Ohlsson A, Shah PS. Paracetamol (acetaminophen) for patent ductus arteriosus in preterm or low-birth-weight infants. Cochrane Database Syst Rev 2015. Doi: 10.1002/14651858.CD010061.pub3

10 Terrin G, Conte F, Oncel MY, et al. Paracetamol for the treatment of patent ductus arteriosus in preterm neonates: a systematic review and meta-analysis. Arch Dis Child Fetal Neonatal Ed 2016;101(02):F127-F136

11 El-Mashad AE, El-Mahdy H, El Amrousy D, Elgendy M. Comparative study of the efficacy and safety of paracetamol, ibuprofen, and indomethacin in closure of patent ductus arteriosus in preterm neonates. Eur J Pediatr 2017;176(02):233-240

12 Härkin P, Härmä A, Aikio O, et al. Paracetamol accelerates closure of the ductus arteriosus after premature birth: a randomized trial. J Pediatr 2016;177:72-77.e2

13 Matsumura S, Oshima A, Fujinuma S, et al. Low-dose intravenous paracetamol for patent ductus arteriosus in indomethacin-resistant or contraindicated preterm infants: three cases reports. AJP Rep 2017;7(04):e230-e233

14 Prescott LF, Illingworth RN, Critchley JA, Stewart MJ, Adam RD, Proudfoot AT. Intravenous N-acetylcystine: the treatment of choice for paracetamol poisoning. BMJ 1979;2(6198):1097-1100

15 Smilkstein MJ, Knapp GL, Kulig KW, Rumack BH. Efficacy of oral Nacetylcysteine in the treatment of acetaminophen overdose. Analysis of the national multicenter study (1976 to 1985). N Engl J Med 1988;319(24):1557-1562

16 Bauer AZ, Kriebel D, Herbert MR, Bornehag CG, Swan SH. Prenatal paracetamol exposure and child neurodevelopment: a review. Horm Behav 2018;101:125-147

17 Oncel MY, Eras Z, Uras N, Canpolat FE, Erdeve O, Oguz SS. Neurodevelopmental outcomes of preterm infants treated with oral paracetamol versus ibuprofen for patent ductus arteriosus. Am J Perinatol 2017;34(12):1185-1189 